\title{
Expression of EMT-Related Factors in Intrahepatic Cholangiolithiasis Associated Cholangiocarcinoma and Its Clinical Significance
}

\author{
Qi Liu', Xiang Rao1, Haixiang Cai' ${ }^{1}$ Juncheng Guo ${ }^{2 *}$ \\ ${ }^{1}$ The Fourth People's Hospital of Haikou, Haikou, China \\ ${ }^{2}$ Hainan General Hospital, Haikou, China \\ Email: ^g2002m@163.com
}

How to cite this paper: Liu, Q., Rao, X., Cai, H.X. and Guo, J.C. (2021) Expression of EMT-Related Factors in Intrahepatic Cholangiolithiasis Associated Cholangiocarcinoma and Its Clinical Significance. Journal of Cancer Therapy, 12, 337-345. https://doi.org/10.4236/jct.2021.126030

Received: May 4, 2021

Accepted: June 5, 2021

Published: June 8, 2021

Copyright $\odot 2021$ by author(s) and Scientific Research Publishing Inc. This work is licensed under the Creative Commons Attribution International License (CC BY 4.0).

http://creativecommons.org/licenses/by/4.0/

\begin{abstract}
In this study, tumor microenvironment and related pathways play an important role in the EMT process of bile duct tumors to analyze the role of EMT in the occurrence and development of cholangiolithiasis associated bile duct carcinoma and investigate the expression of EMT in intrahepatic cholangiolithiasis associated cholangiocarcinoma and its clinical significance.
\end{abstract}

\section{Keywords \\ EMT, The Liver, Cancer, Organization}

\section{Introduction}

Epithelial-mesenchymal transition (EMT) is a process in which epithelial cells undergo epithelial-mesenchymal transition to mesenchymal transition when stimulated by external signals, resulting in loss of cell polarity and cell-cell adhesion, and enhancement of cell mobility and migration. EMT is believed to be a major mechanism by which cancer cells become migratory and invasive, enabling the dissemination of cancer cells. EMT plays a very important role in physiological processes such as embryo development, organ generation and wound healing [1]. The most significant molecular event in EMT process is decreased expression of E. adherin and increased expression of E-cadherin/Vimentin. The transcription factors that induced EMT are mainly SNML, Slug and Twig [2]. Studies have shown that EMT in tumor cells is regulated by some important signal transduction pathways, including TGF- $\beta$, Notch, Hedgehog and EGF sig- 
nal transduction pathways [3]. TGF- $\beta$ induces EMT mainly involves some signaling pathways related to cell movement and apoptosis, such as the RHO. 1IKE GTPase pathway, the P13K/Akt pathway or the MAPK pathway [4]. In recent years, more and more experimental evidence has shown that EMT process is closely related to tumor progression, especially tumor cell infiltration and metastasis. At the front end of tumor tissue invasion, tumor cells receive signals from tumor cells, tumor-associated fibroblasts, and inflammatory cells, and transform from epithelial cells to cytoplasmic cells, thus leaving the primary site and infiltrating into local tissues. The stromal tumor cells that have separated from the primary site travel through the hematological or lymphoid pathways to distant tissues and continue to infiltrate surrounding tissues. After reaching the distant site, Mesenchymal Epithelial Transition (MET) occurs and begins to grow, leading to the formation of metastatic lesions [5] [6]. EMT mainly plays an important role in the early stage of tumor cell invasion and metastasis, which alternately regulates cell adhesion, cell migration and cell invasion. In this process, the composition of Extra Cellular Matrix (ECM) (including proteoglycan, collagen, fibrinogen, elastin, laminin, etc.), Cell Adhesion Molecules (CAMs; Ig superfamily, Integrin, Cadherin, Selectin, and CD44) play a key role in mediating cellcell and cell-matrix interactions [7]. Intigrin is a membrane protein that mediates cellular and extracellular matrix interactions. This membrane protein consists of different $\mathrm{N}$ subunits and $\mathrm{D}$ subunits to form 24 heterodimer forms, each of which binds to different ligands to mediate the corresponding signal transduction process [8]. Degradation of extracellular matrix (ECM) is critical for tumor cells to invade surrounding tissues. Matrixmetalloproteinases (MMPs) are a broad spectrum of proteases secreted extracellular, degrading substrates including collagen, plasminogen, elastin, fibrinogen, laminin, etc. Among them, MMP-2 and MMP-9 have strong degradation activity on IV type collagen, the main component of basement membrane, so they are closely related to tumor metastasis. Intigrin can regulate the expression and secretion of MMPs through the MAPK pathway, and ultimately affect the invasion and metastasis of tumors [9]. E-cadherin mainly mediates the adhesion between homologous cells in epithelial tissue, and transmits signals between epithelial cells. In colon cancer, the down-regulated expression of E-cadherin is conducive to the separation of tumor cells from the primary tumor and invasion of surrounding tissues [10]. In glioma, prostate cancer, and breast cancer, downregulation of E-cadherin is accompanied by up-regulation of $\mathrm{N}$-cadherin and cadherin-11 expression, both of which are mesenchymal Cadherin. This transformation of Cadherin may facilitate the invasion of tumor cells to the surrounding environment. CD44 is a transmembrane glycoprotein, which acts as a receptor for hyaluronic acid and ECM components, and the signal is finally transmitted to the nucleus through Rho/Ras and tyrosine protein kinase pathways [11]. The tumor cells are stimulated by external molecules such as ECM components or MMPs to activate the intracellular Rho signaling pathway, and the cytoskeleton component Actin causes the cell membrane to extend out of the pseudopod through alternating polyme- 
rization and depolymerization, promoting cell forward migration [12].

\section{EMT-Related Molecular Changes Are Present in Adjacent Tissues}

To date, the vast majority of cancer-related EMT research has focused on tumor cells and advanced tumor progression. Can EMT also play a role in the early stages of tumorigenesis, such as atypical hyperplasia or carcinoma in situ, and in paracancerous tissues where there is no significant histomorphologic abnormality? Several recent studies offer a preliminary answer. In the study of pancreatic cancer induced by transgenic mice, Rhim et al. [13] detected different stages of pancreatic cancer, and found that changes in EMT-related molecular markers (Zebl, Slug, Snaill, etc.) could be detected in the precancerous lesion "PANLN "(carcinoma in situ). Also in the "PANLN" stage, a part of pre-malignant pancreatic epithelial cells separated from the lesion site and entered the peripheral circulating blood through EMT, called circulating pancreatic epithelial cells (CPCs) in Chinese. These CPCs had the characteristics of tumor stem cells and could form tumors when injected into nude mice. It was also found that inflammatory response may play an important role in EMT and early spread of epithelial cells in "PanIN" lesions. This study is the first to demonstrate that pancreatic cancer can develop distant metastases early in its development (carcinoma in situ) and is significantly associated with the EMT process. According to the theory of regional carcinogenesis, due to the existence of "field effect", adjacent tissues with normal histological morphology also have some molecular changes similar to those of tumor tissues. Secondly, various cytokines (VEGFA, TGF $\beta$, TNF- $\alpha$, etc.) released by tumor cells and tumor microenvironment may also exert effects on adjacent normal tissues. Analysis results from another study showed that these gene expression changes may be associated with EMT, because EMT-related marker molecules (Vimentin, Snail, $\alpha$-SMA and TGF $\beta$ ) were positively expressed in breast ductal epithelial cells of adjacent cancer tissues [14].

Several studies have found that the expressions of interstitial markers such as Vimentin and S100 and related transcription factors are also increased in bile duct epithelial tumors, while the expressions of epithelial markerse. Adherin and EpCAM are downregulated. This phenomenon is obviously associated with the aggressive biological behavior of tumors. Araki et al. [15] found that E-cadherin and $\mathrm{N}$-cadherin conversion are risk factors for poor prognosis in patients with cholangiocarcinoma. Nitta et al. [16] found that the down-regulated expression of E-cadherin and up-regulated expression of Vimentin significantly affected the postoperative survival time of bile duct carcinoma.

\section{Study on the Related Pathways Affecting the EMT Process of Bile Duct Cell Carcinoma}

A number of studies have found that multiple pathways regulate the EMT process 
of bile duct cell carcinoma. Notchl expression was significantly upregulated in the carcinoma tissues of intrahepatic bile duct cell carcinoma. With the upregulation of Notchl, the invasion ability of tumor cells was enhanced, and tumor cells appeared EMT phenomenon. Inhibitory expression of Notchl will inhibit the migration of bile duct cancer cells. Overexpression of Notchl will induce downregulation of E-CDherin expression, up-regulation of $\alpha$-SMA and Vimentin, change of tumor cell phenotype and enhance invasion ability. Shuang et al. [17] found that in bile duct tumor cells, high expression of TGF- $\beta 1$ can increase the expression of stromal markers of bile duct tumor cells, enable tumor cells to obtain stromal cell characteristics and enhance the metastasis ability. High expression of TGF- $\beta 1$ is a risk factor for poor prognosis, and it is speculated that TGF$\beta$-mediated EMT process is involved in the formation of tumor stem cells. TGF$\beta$ signaling pathway involves a variety of genes, proteins and transcription factors [18] [19]. $\beta$-catenin in the Wnt pathway is associated with tumor EMT process. $\beta$-catenin connects to E-cadherin, actin, and $\alpha$-catenin to form a complex that mediates cell-to-cell adhesion. When the degradation of the synthesized B-catenin is blocked, it can enter the nucleus and synergise with related transcription factors to activate Slug and other Wnt target genes. Chen et al. [20] compared the expression of FJ (Wnt, $\beta$-catenin) associated with the Wnt pathway in patients with intrahepatic cholangiocellular carcinoma, hilar cholangiocarcinoma and congenital bile duct cyst by immunohistochemical method, and then analyzed it in combination with tumor lymph node metastasis and postoperative survival of patients. Boulter et al. [21] found that Wnt pathway was highly activated in cholangiocellular carcinoma, and inhibition of Wnt pathway could inhibit the proliferation and induce the apoptosis of tumor cells.

\section{Tumor Microenvironment and a Variety of Cells and Cytokines Can Promote the EMT Process of Bile Duct Cell Carcinoma}

Many cytokines and growth factors can promote the EMT process of cholangiocarcinoma, including TGF- $\beta$ 1, Tumor Necrosis Factor $\alpha$, Epidermal GrowthFactor (EGF), interleukin. 6 (IL-6), and Notchl play an important role in tumor EMT process after being released by various cells in tumor microenvironment [22] [23]. In addition, para-tumor active matrix also plays an important role in the process of tumor EMT. Para-tumor active matrix is a highly specific stromal component, including a variety of cell types, such as tumor-associated fibroblasts and tumor-associated phagocytes [24] [25]. Tumor-associated fibroblasts and tumor-associated phagocytes can secrete a number of cytokines and growth factors, such as TGF- $\beta$, TNFA, EGF, IL- 6 , etc. These factors trigger the EMT process of bile duct tumor cells, and the loss of epithelial biomarkers such as $\mathrm{E}$. adherin enhances the invasion ability of tumor cells. Techasen et al. [26] found that activated macrophages can secrete a variety of cytokines (including TGF- $\beta 1$, TNFA, EGF and IL-6), and when these cytokines were added to cultured bile duct cancer cell lines in vitro, the bile duct cancer cells developed EMT pheno- 
menon, and the expressions of E-cadherin and CKL9 were significantly reduced. The expression of S100 and Vimentin was up-regulated, and the cell invasion ability was enhanced. Similarly, Okamoto et al. [27] found that tumor-associated fibroblasts can secrete SDF- $\beta 1$ under activation state, thereby promoting the down-regulation of E-cadherin in bile duct cancer cells, up-regulation of interstitial markers, and initiation of EMT process. Zhao et al. [28] found that a similar phenomenon also exists in breast cancer cells. Ang II (Ang II) can activate the P13K signaling pathway first, and then activate the EMT process of breast cancer cells through the NF. In addition, Kurashige et al. [29] found that tumor-associated fibroblasts can induce the EMT process of gastric cancer cells through DNA methylation of miR200b and promote gastric cancer metastasis. Local hypoxic environment of tumor cells can also promote the occurrence of EMT process of tumor cells. Hypoxic environment may promote the secretion of adrenal medullohormone (ADM) by bile duct tumor cells, and the overexpression of ADM can promote the occurrence of EMT, thus enhancing the invasion ability of tumor cells [30]. This phenomenon also exists in tumor cells of other tissues. Zhu et al. [31] found in their study that ADM produced in hypoxia environment can promote the EMT process of proximal convoluted tubular epithelial cells.

\section{Autophagy Plays an Important Role in the EMT Process of Bile Duct Cell Carcinoma}

Autophagy is a classic phenomenon in the process of intracellular energy metabolism and cellular self-renewal, which plays an important role in the process of biological growth and maintaining the stability of the organism's environment. Existing studies have found that autophagy plays an important role in the process of tumor proliferation, invasion and energy metabolism, and autophagy can be induced by a variety of factors, such as hypoxia environment and nutrient deficiency state [32]. Catalano et al. [33] found that in malignant glioma, nutritional deprivation can lead to upregulation of Slig and Snial in tumor cells, thereby reducing the expression of E-cadherin and increasing the expression of interstitial markers $\mathrm{N}$-cadherin and R-cadherin, thus promoting the EMT process of tumor cells. Autophagy is also found in bile duct cancer cell lines. Studies have found that in a nutritional deprivation environment, the expression of autophagy-related protein Ambral is significantly increased, and Ambral can positively regulate the expression of the transcription factor Slug, thereby promoting the down-regulation of E-cadherin and up-regulation of Vimentin in bile duct cell carcinoma. However, EMT processes in tumor cells after transfection with an inhibitor of Ambral.

Therefore, autophagy may promote the occurrence of EMT and the infiltration of tumor cells, which is an adverse factor for the prognosis of bile duct cell carcinoma [34].

\section{Conclusions}

In recent years, the incidence of cholangiocarcinoma has increased significantly, 
especially intrahepatic cholangiocarcinoma. Many factors have been confirmed to be associated with the incidence of cholangiocarcinoma [35], such as cholangiolithiasis, congenital biliary cyst, primary sclosing cholangitis, viral hepatitis, alcohol consumption, metabolic syndrome, schistosomiasis liver disease, etc. However, the pathogenesis of cholangiocarcinoma is still not clear at present [36] [37]. EMT has been found to be involved in the process of metastasis and invasion of a variety of tumors, as well as in highly aggressive cholangiocarcinoma. EMT process of bile duct cell carcinoma involves multiple pathways, the expression of numerous cytokines and growth factors, and various miRNAs. In addition, tumor microenvironment and autophagy also play an important role in the EMT process of bile duct carcinoma [38] [39] [40].

The migration and metastasis of malignant tumors are the important characteristics of malignant tumors. The process of tumor metastasis is a complex process involving many signaling pathways. EMT has been found to be involved in metastasis and invasion of a variety of tumors, as well as in cholangiocarcinoma, a highly aggressive tumor. The EMT process of bile duct cell carcinoma involves multiple pathways, the expression of numerous cytokines and growth factors, and various miRNAs. In addition, tumor microenvironment and autophagy also play an important role in the EMT process of bile duct carcinoma. To further study the EMT mechanism of bile duct tumors, it is of great significance to find potential therapeutic targets for targeted therapy of bile duct cell carcinoma and to improve the prognosis of bile duct cell carcinoma.

\section{Foundation Project}

This study was supported by Hainan Provincial Basic (Project No. 819MS144).

\section{Conflicts of Interest}

The authors declare no conflicts of interest regarding the publication of this paper.

\section{References}

[1] Thiery, J.P., Acloque, H., Huang, R.Y., et al. (2009) Epithelial-Mesenchymal Transitions in Development and Disease. Cell, 139, 871-890.

https://doi.org/10.1016/j.cell.2009.11.007

[2] Wu, Y. and Zhou, B.P. (2008) New Insights of Epithelial-Mesenchymal Transition in Cancer Metastasis. Acta Biochimica et Biophysica Sinica (Shanghai), 40, 643-650. https://doi.org/10.1111/j.1745-7270.2008.00443.x

[3] Moustakas, A. and Heldin, C.H. (2007) Signaling Networks Guiding Epithelial-Mesenchymal Transitions during Embryogenesis and Cancer Progression. Cancer Science, 98, 1512-1520. https://doi.org/10.1111/j.1349-7006.2007.00550.x

[4] Lee, B.S., Park, E.C., Park, S.W., et al. (2015) Hepatitis B Virus Infection, Diabetes Mellitus, and Their Synergism for Cholangiocarcinoma Development: A CaseControl Study in Korea. World Journal of Gastroenterology, 21, 502-510. https://doi.org/10.3748/wjg.v21.i2.502

[5] Chaffer, C.L., Thompson, E.W. and Williams, E.D. (2007) Mesenchymal to Epi- 
thelial Transition in Development and Disease. Cells Tissues Organs, 185, 7-19. https://doi.org/10.1159/000101298

[6] Geiger, T.R. and Peeper, D.S. (2009) Metastasis Mechanisms. Biochimica et Biophysica Acta, 1796, 293-308. https://doi.org/10.1016/j.bbcan.2009.07.006

[7] Guglielmi, A., Ruzzenente, A., Valdegamberi, A., et al. (2014) Hepatolithiasis-Associated Cholangiocarcinoma: Results from a Multi-Institutional National Database on a Case Series of 23 Patients. European Journal of Surgical Oncology, 40, 567-575. https://doi.org/10.1016/j.ejso.2013.12.006

[8] Dowling, P., Walsh, N. and Clynes, M. (2008) Membrane and Membrane-Associated Proteins Involved in the Aggressive Phenotype Displayed by Highly Invasive Cancer Cells. Proteomics, 8, 4054-4065. https://doi.org/10.1002/pmic.200800098

[9] Chenette, E.J. (2009) Cancer: A Ras and NF-kappaB pas de deux. Nature Reviews Drug Discovery, 8, 932. https://doi.org/10.1038/nrd3060

[10] Restucci, B., Martano, M., et al. (2009) Expression of E-cadherin, beta-catenin and APC Protein in Canine Colorectal Tumours. Anticancer Research, 29, 2919-2925.

[11] Bourguignon, L.Y., Peyrollier, K., Xia, W., et al. (2008) Hyaluronan-CD44 Interaction Activates Stem Cell Marker Nanog, Stat-3-Mediated MDR1 Gene Expression, and Ankyrin-Regulated Multidrug Efflux in Breast and Ovarian Tumor Cells. Journal of Biological Chemistry, 283, 17635-17651.

https://doi.org/10.1074/jbc.M800109200

[12] Jemal, A., Bray, F., Center, M.M., et al. (2011) Global Cancer Statistics. CA: A Cancer Journal for Clinicians, 61, 69-90. https://doi.org/10.3322/caac.20107

[13] Rhim, A.D., Mirek, E.T., Aiello, N.M., et al. (2012) EMT and Dissemination Precede Pancreatic Tumor Formation. Cell, 148, 349-361.

https://doi.org/10.1016/j.cell.2011.11.025

[14] Trujillo, K.A., Heaphy, C.M., Mai, M., et al. (2011) Markers of Fibrosis and Epithelial to Mesenchymal Transition Demonstrate Field Cancerization in Histologically Normal Tissue Adjacent to Breast Tumors. International Journal of Cancer, 129, 1310-1321. https://doi.org/10.1002/ijc.25788

[15] Araki, K., Shimura, T., Suzuki, H., et al. (2011) E/N-Cadhefin Switch Mediates Cancer Progression via TGF-Beta-Induced Epithelial-to-Mesenchymal Transition in Extrahepatic Cholangiocarcinoma. British Journal of Cancer, 105, 1885-1893. https://doi.org/10.1038/bjc.2011.452

[16] Nitta, T., Mitsuhashi, T., Hatanaka, Y., et al. (2014) Prognostic Significance of Epithelial-Mesenchymal Transition-Related Markers in Extrahepatic Cholangiocarcinoma: Comprehensive Immunohistochemical Study Using a Tissue Microarray. British Journal of Cancer, 111, 1363-1372. https://doi.org/10.1038/bjc.2014.415

[17] Shuang, Z.Y., Wu, W.C., Xu, J., et al. (2014) Transforming Growth Factor-BetalInduced Epithelial-Mesenchymal Transition Generates ALDH-Positive Cells with Stem Cell Properties in Cholangiocarcinoma. Cancer Letters, 354, 320-328. https://doi.org/10.1016/j.canlet.2014.08.030

[18] Willis, B.C. and Borok, Z. (2007) TGF-Beta-Induced EMT: Mechanisms and Implications for Fibrotic Lung Disease. American Journal of Physiology-Lung Cellular and Molecular Physiology, 293, L525-L534. https://doi.org/10.1152/ajplung.00163.2007

[19] Acloque, H., Adams, M.S., Fishwick, K., et al. (2009) Epithelial-Mesenchymal Transitions: The Importance of Changing Cell State in Development and Disease. Journal of Clinical Investigation, 119, 1438-1449. https://doi.org/10.1172/JCI38019

[20] Chen, W., Liang, J., Huang, L., Huang, L., Cai, J., et al. (2016) Characterizing the 
Activation of the Wnt Signaling Pathway in Hilar Cholangiocarcinoma Using a Tissue Microarray Approach. European Journal of Histochemistry, 60, 2536. https://doi.org/10.4081/ejh.2016.2536

[21] Boulter, L., Guest, R.V., Guest, R.V., Kendall, T.J., Kendall, T.J., Wilson, D.H., et al. (2015) WNT Signaling Drives Cholangiocarcinoma Growth and Can Be Pharmacologically Inhibited. Journal of Clinical Investigation, 125, 1269-1285. https://doi.org/10.1172/JCI76452

[22] Li, M. and Deng, F. (2015) The Role of miR-200c in Tumor Diagnosis, Metastasis and Drug Resistance. International Journal of Oncology, 42, 602-604.

[23] Peinado, H. and Olmedad, C.A. (2007) Snail, ZEB and bHLH Factors in Tumour Progression: An Alliance against the Epithelial Phenotype? Nature Reviews Cancer, 7, 415-428. https://doi.org/10.1038/nrc2131

[24] Bn, S. and Na, B. (2016) Role of EMT in Metastasis and Therapy Resistance. Journal of Clinical Medicine, 5, 17. https://doi.org/10.3390/jcm5020017

[25] Claperon, A., Mergey, M., Bouldoires, T.H.N., et al. (2014) EGF/EGFR Axis Contributes to the Progression of Cholangiocarcinoma through the Induction of an Epithelial-Mesenchymal Transition. Journal of Hepatology, 61, 325-332. https://doi.org/10.1016/j.jhep.2014.03.033

[26] Techasen, A., Loilome, W., et al. (2012) Cytokines Released from Activated Human Macrophages Induce Epithelial Mesenchymal Transition Markers of Cholangiocarcinoma Cells. Asian Pacific Journal of Cancer Prevention, 13, 115-118.

[27] Okamoto, K., Tajima, H., Nakanuma, S., et al. (2012) Angiotensin II Enhances Epithelial-to-Mesenchymal Transition through the Interaction between Activated Hepatic Stellate Cells and the Stromal Cell-Derived Factor-1/CXCR4 Axis in Intrahepatic Cholangiocarcinoma. International Journal of Oncology, 41, 573-582. https://doi.org/10.3892/ijo.2012.1499

[28] Zhao, Y.B., Wang, H.B., Li, X.L., et al. (2014) Ang II-AT1R Increases Cell Migration through P13K/AKT and NF-kappa B Pathways in Breast Cancer. Journal of Cellular Physiology, 229, 1855-1862. https://doi.org/10.1002/jcp.24639

[29] Kurashige, J., Mima, K., Sawada, G., et al. (2015) Epigenetic Modulation and Repression of miR-200b by Cancer-Associated Fibroblasts Contribute to Cancer Invasion and Peritoneal Dissemination in Gastric Cancer. Carcinogenesis, 36, 133-141. https://doi.org/10.1093/carcin/bgu232

[30] Zhou, C., Zheng, Y., Li, L., et al. (2015) Adrenomedullin Promotes Intrahepatic Cholangiocellular Carcinoma Metastasis and Invasion by Inducing Epithelial Mesenchymal Transition. Ontology Reports, 34, 610-616.

https://doi.org/10.3892/or.2015.4034

[31] Zhu, T.C., Yang, J., Liu, X.O., et al. (2015) Hypoxia-Inducible Adrenomedullin Ameliorates the Epithelial-to-Mesenchymal Transition in Human Proximal Tubular Epithelial Cells. Molecular Medicine Reports, 11, 3760-3766. https://doi.org/10.3892/mmr.2015.3189

[32] Zhang, M.N., Tang, Z.J. and Chen, L. (2014) The Role of Autophagy-Apoptosis Pathway in Tumor Targeted Therapy. Journal of Oncology, 5, 413-418.

[33] Catalano, M., D’alessandro, G., Lepore, F., et al. (2015) Autophagy Induction Impairs Migration and Invasion by Reversing EMT in Glioblastoma Cells. Molecular Oncology, 9, 1612-1625. https://doi.org/10.1016/j.molonc.2015.04.016

[34] Nitta, T., Sato, Y., Ren, X.S., et al. (2014) Autophagy May Promote Carcinoma Cell Invasion and Correlate with Poor Prognosis in Cholangiocarcinoma. International Journal of Clinical and Experimental Pathology, 7, 4913-4921. 
[35] Wu, Z.F., Yang, N., Li, D.Y., et al. (2013) Characteristics of Intrahepatic Cholangiocarcinoma in Patients with Hepatitis B Virus Infection: Clinicopathologic Study of Resected Tumours . Journal of Viral Hepatitis, 20, 306-316. https://doi.org/10.1111/jvh.12005

[36] Su, X.C., Pang, S.J., Yang, N., et al. (2015) Clinical Characteristics and Prognosis of Intrahepatic Cholangiocellular Carcinoma Associated with Intrahepatic Cholangiolithiasis. Journal of Jiangsu University: Medical Science, N. 3, 241-246.

[37] Shimada, K., Sano, T., Sakamoto, Y., et al. (2007) Outcomes of the Mass-Forming plus Periductal Infiltrating Types of Intrahepatic Cholangiocarcinoma: A Comparative Study with the Typical Mass-Forming Type of Intrahepatic Cholangiocarcinoma. World Journal of Surgery, 31, 2016-2022.

https://doi.org/10.1007/s00268-007-9194-0

[38] Xu, J., Sasaki, M., Harada, K., et al. (2011) Intrahepatic Cholangiocarcinoma Arising in Chronic Advanced Liver Disease and the Cholangiocarcinomatous Component of Hepatocellular Cholangiocarcinoma Share Common Phenotypes and Cholangiocarcinogenesis. Histopathology, 59, 1090-1099.

https://doi.org/10.1111/j.1365-2559.2011.04058.x

[39] Aishima, S., Kuroda, Y., Nishihara, Y., et al. (2007) Proposal of Progression Model for Intrahepatic Cholangiocarcinoma: Clinicopathologic Differences between Hilar Type and Peripheral Type. American Journal of Surgical Pathology, 31, 1059-1126. https://doi.org/10.1097/PAS.0b013e31802b34b6

[40] Nakanuma, Y., Miyata, T. and Uchida, T. (2016) Latest Advances in the Pathological Understanding of Cholangiocarcinomas. Expert Review of Gastroenterology \& Hepatology, 10, 113-140. https://doi.org/10.1586/17474124.2016.1104246 